\title{
Rise in life expectancy is marred by widening inequality gap
}

In this News article by Ingrid Torjesen there are some errors about inequality in men's life expectancy (BMJ 2012;344:e1141, doi:10.1136/bmj.e1141). The final paragraph should have said that the largest rise in inequality in life expectancy in men was in West Berkshire and was two years [not Merton and 1.5 years, as published] and that the largest decrease in inequality for men was in Kensington and Chelsea [not Tower Hamlets].

Cite this as: BMJ 2012;343:e1390

๑ BMJ Publishing Group Ltd 2012 